\title{
THERMOELECTRICAL PROCESSES SIMULATION IN RESISTIVE SUPERCONDUCTING FAULT CURRENT LIMITER
}

\author{
Dmytro Fedasyuk, Pavlo Serdyuk \\ Lviv Polytechnic National University, S. Bandery Str., 12, Lviv, 79013, UKRAINE \\ e-mail: fedasyuk@polynet.lviv.ua
}

\begin{abstract}
Simulations of superconducting fault current limiter (SFCL) include modelling of thermal and electrical behaviour of the limiter. In this paper the mathematical model for the analysis of thermoelectrical processes in resistive superconducting fault current limiter for microelectronics devices protection is presented. An effective and fast halfanalytical computer simulation of the model is presented. Simulation results are presented.
\end{abstract}

Keywords: Resistive superconducting fault current limiter, thermal modeling, thermoelectrical processes.

\section{INTRODUCTION}

The need for fault current limiters (FCL) for microelectronic devices is associated with the continuous growth and interconnection of modern power systems and increase of dispersed generation facilities, which result in progressive increase in the short circuit capacity far beyond their original design capacity.

Conventional methods for current limiting incur losses during the normal system operation, some of them need replacement after short circuit, other equipment is rather expensive and reduces the operational flexibility and system stability.

Superconductivity brings new solution to the problem of short circuit protection of microelectronic devices from critical current. Resistive superconducting fault current limiter (SFCL) meets the main requirements of current limiter devices - they protect system from failure and do not influence the electric system during normal system operation. Besides, resistive superconducting fault current limiters have a number of advantages over traditional fault current limiter devices: rapid response, automatic recovery after fault current, modularity and simple architecture expansion of protection devices on SFCL.

In order to design SFCL for practical applications, knowledge of SFCL parameters such as maximum load, response time and thermal recovery time is essential.

On the other hand, the electromagnetic and thermal response of a SFCL to a fault involves very high voltages and currents at very short times, and is therefore a formidable challenge for experiments. Computer simulations are obviously helpful, since they allow research on arbitrary time scales and power level.

A lumped network model of resistive superconductor limiter based on a double noninductive spiral is presented by Petranovic and Miri [1]. To simulate the resonant behavior of the limiter, electric and magnetic fields are assigned to an electric network consisting of lumped elements. Each winding turn of a module and coupling between winding were modeled by specific element.

In papers [2, 3] the electrical, thermal and mechanical model of current limiting device with quench and melt growth bulk superconductor is presented. Electrical circuit analysis for simulation of current sharing is coupled at each time step with the finite difference method to calculate the temperature distribution inside the device. It was concluded that magnitude of mechanical stress of limiting device is less than allowable level of each model device.

Computer model for the simulation of electromagnetic and thermal response of superconducting fault current limiter was presented in [4]. In this paper the influence of cryogen boil-off parameters and height of superconductor bar was investigated.

Another problem of high-temperature superconductors is the thermal instability near planar defects or low-angle boundaries. Hot spots near small defects and overheating may cause transition from superconducting to normal state even at low current density $[5,6]$. Local exceeding of the 
temperature due to Joule heating near contacts may destroy superconductivity state. Simulation of thermal processes in superconductor due to metalsuperconductor junctions was presented in papers $[7$, $8,9]$.

Thermoelectrical processes in superconductor are described by nonlinear differential equations. Numerical simulation of these processes causes the instability of equation solution [4].

Our simulation uses the analytical approach to calculate thermal field in SFCL, which allows to obtain accurate solution of heat distribution in resistive fault current limiter.

\section{THERMOELECTRICAL MODEL FORMALIZATION}

Schematic representation of SFCL construction is shown in Fig 1. The current limiting device consists of a sapphire substrate, high-temperature film YBCO and gold deposited in superconductor for the contacts. Superconductor element YBCO is cooled by liquid nitrogen to keep the temperature level below the critical.

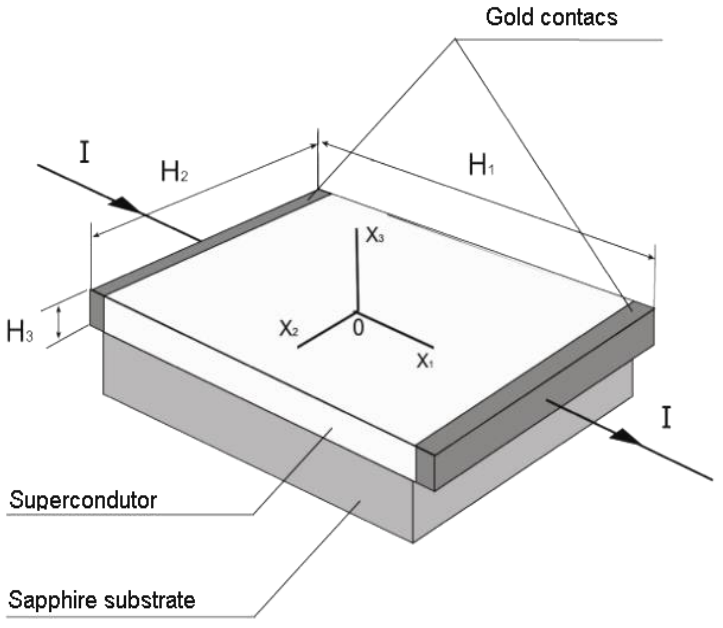

Fig. 1 - Construction of resistive SFCL element.

Superconducting film can come to resistive state due to internal thermal power losses that depend on temperature and current. Three thermoelectrical regimes of SFCL are considered:

- Normal regime, when the element is in superconducting state: current density and temperature is lower then critical $i_{n}<i_{c}, T<T_{c}$, there are no power losses in superconductor;

- Critical regime, when the current density exceeds superconductor critical current density $i_{k}>i_{c}$ and superconductor comes to resistive state. Assume that time of current fault is small and the current in critical regime is homogeneous;

- $\quad$ Restoring regime, when current come back to normal on SFCL after some time in critical regime. Superconductor temperature in this regime is going down to temperature of liquid nitrogen.

Assumption and analysis of boundary conditions:

1. Determine the system of coordinate with axis parallel to the superconductor borders and with a center in the middle of the bottom superconductor element (see Fig.1). Thermal distribution is symmetric for planes $x_{1}=0, x_{2}=0$.

2. Assume that sapphire substrate does not participate in thermal and electrical processes. On the edge of sapphire thermal flow is equal to zero.

3. Density of heat dissipation at the contact equals $i^{2} \rho_{c} H_{2} H_{3}$, where $\rho_{c}$ - is the electrical resistance of contact surface. Heat flow from the contact to liquid nitrogen equals $\beta_{c}\left(T-T_{0}\right) H_{2} H_{3}$, and heat flow from contact to superconductor equals $\lambda_{c} \frac{\partial T}{\partial x_{1}} H_{2} H_{3}$, where $\beta_{c}$ - coefficient of cooling gold contacts by liquid nitrogen, $\lambda_{c}$ - contact thermal resistance, $T_{0}$ - temperature of liquid nitrogen. In our approach we disregard heating of contact and assume thermal balance in the contact area:

$$
i^{2} \rho_{c}=\beta_{c}\left(T-T_{0}\right)+\lambda_{c} \frac{\partial T}{\partial x_{1}}
$$

Thermal processes in superconductor are described by nonlinear equation:

$$
c \rho \frac{\partial T}{\partial t}=\lambda \nabla T+\rho(i, T) i^{2}
$$

here $f(i, T)$ - thermal heating in superconductor, $c$ and $\lambda$ - thermal capacity and thermal conductance of superconducting material, $\rho$ - density of material.

According to the assumption about thermoelectrical regimes, electrical resistance in superconductor can be described by the following function:

$$
\rho(i, T)= \begin{cases}0, & \left(i<i_{c}\right) \wedge\left(T<T_{c}\right) \\ \rho_{n}+\alpha_{\rho}\left(T-T_{c}\right), & \left(i>i_{c}\right) \vee\left(T>T_{c}\right)\end{cases}
$$

Here $T_{c}$ - critical temperature of superconductor, $i_{c}(T)$ - critical current density of superconductor, $\rho_{n}$ - electrical resistance of superconductor material in resistive state, $\alpha_{\rho}$ - coefficient that describes influence of temperature at electrical resistance. 


$$
\begin{aligned}
& \text { Define } a=\frac{\lambda}{c \rho}, b_{k}=\frac{\alpha_{\rho}\left(i_{c}\right)^{2}}{c \rho}, b_{n}=\frac{\alpha_{\rho}\left(i_{n}\right)^{2}}{c \rho}, \\
& c_{k}=\frac{\left(i_{c}\right)^{2}}{c \rho}\left(\rho_{c}-\alpha_{\rho} T_{c}\right), c_{n}=\frac{\left(i_{n}\right)^{2}}{c \rho}\left(\rho_{c}-\alpha_{\rho} T_{c}\right) .
\end{aligned}
$$

In normal regime will temperature distribution is stationary. Thermal characteristics are constant in this regime, and from equation (2) we obtain:

$$
\nabla T_{n}(x)=0
$$

where $T_{n}(x)$ - temperature at point $x=\left(x_{1}, x_{2}, x_{3}\right)^{T}$ in normal regime.

At the beginning of critical regime temperature distribution equals to $T_{n}(x)$. Denote temperature distribution in critical regime with $T_{k}(x, t)$, then

$$
\begin{aligned}
T_{k}(x, 0) & =T_{n}(x) ; \\
& \frac{\partial T_{k}}{\partial t}=a^{2} \nabla T_{k}+b_{k} T+c_{k},
\end{aligned}
$$

here $i_{k}$ is the density of current in critical regime.

In restoring regime, thermal processes will be described by equation:

$$
\frac{\partial T_{r}}{\partial t}=a^{2} \nabla T_{r}+f(T), t \geq \tau_{k}
$$

Here $T_{r}\left(x, \tau_{k}\right)$ - temperature distribution in restoring regime, $f(T)=\left\{\begin{array}{l}0, T<T_{c} \\ b_{n} T+c_{n}, T>T_{c}\end{array}\right.$

At the beginning of restoring regime distribution of the temperature is $T_{k}\left(x, \tau_{k}\right)$, where $\tau_{k}$ is fault current time.

$$
T_{r}\left(x, \tau_{k}\right)=T_{k}\left(x, \tau_{k}\right) .
$$

Supplement model with boundary conditions.

Superconductor element is cooled by liquid nitrogen:

$$
\begin{aligned}
& \left.\left(\frac{\partial T}{\partial x_{2}}-\beta\left(T-T_{0}\right)\right)\right|_{x_{2}=\frac{H_{2}}{2}}=0 ; \\
& \left.\left(\frac{\partial T}{\partial x_{3}}-\beta\left(T-T_{0}\right)\right)\right|_{x_{3}=H_{3}}=0,
\end{aligned}
$$

where $\beta$ - coefficient of cooling YBCO of liquid nitrogen.

Condition of symmetry:

$$
\left.\left(\frac{\partial T}{\partial x_{i}}\right)\right|_{x_{i}=0}=0, \mathrm{i}=1,2 .
$$

According to boundary condition assumption for sapphire substrate:

$$
\left.\left(\frac{\partial T}{\partial x_{3}}\right)\right|_{x_{3}=0}=0
$$

At the contacts metal-superconductor:

$$
\left.\left(\lambda_{c} \frac{\partial T}{\partial x_{1}}+\beta_{c} T+c_{c}\right)\right|_{x_{1}=\frac{H_{1}}{2}}=0
$$

where $c_{k}=-\beta_{c} T_{0}-i^{2} \rho_{c}$.

\section{SOLUTION OF MATHEMATICAL MODEL OF THERMOELECTRICAL PROCESSES IN SFCL}

In normal regime thermal distribution appears as:

$$
T_{n}(x)=T_{0}+\sum_{i, j} C_{i j}^{n} \varphi_{i j}^{n}(x),
$$

where

$$
\varphi_{i j}^{n}\left(x_{1}\right)=\operatorname{ch}\left(m_{i j} x_{1}\right) \cos \left(k_{i}^{2} x_{2}\right) \cos \left(k_{j}^{3} x_{3}\right)
$$

$C_{i j}^{n}=\frac{2}{\left(\sin \left(k_{i}^{2} H_{2}\right)+k_{i}^{2} H_{2}\right)\left(\sin \left(2 k_{j}^{3} H_{3}\right)+2 k_{j}^{3} H_{3}\right)} \times$

$\times \frac{4\left(\beta_{c} T_{0}+c_{c}\right) \sin \left(k_{i}^{2} \frac{H_{2}}{2}\right) \sin \left(k_{j}^{3} H_{3}\right)}{\left(\lambda_{c} m_{i j} \operatorname{sh}\left(m_{i j} \frac{H_{1}}{2}\right)+\beta_{c} c h\left(m_{i j} \frac{H_{1}}{2}\right)\right)}$

$k_{i}^{2}, \mathrm{i}=1,2 \ldots$ - solutions of equation:

$k_{i}^{2}+\operatorname{ctg}\left(k_{i}^{2} \frac{H_{2}}{2}\right)=0$,

$k_{j}^{3}, \mathrm{j}=1,2 \ldots-$ solutions of equation:

$k_{j}^{3}+\operatorname{ctg}\left(k_{j}^{3} H_{3}\right)=0$,

$m_{i j}=\sqrt{\left(k_{i}^{2}\right)^{2}+\left(k_{j}^{3}\right)^{2}}$. 
Solution of thermal equation was found for critical regime:

$$
T_{k}(x, t)=T_{n}(x)+\sum_{i, j, m} C_{m i j}^{k} \varphi_{m i j}^{k}(x, t),
$$

where $k_{m}^{1}, \mathrm{~m}=1,2$ - solutions of equation

$$
\begin{aligned}
& k_{m}^{1}=\frac{\beta_{c}}{\lambda_{c}} \operatorname{ctg}\left(k_{m}^{1} \frac{H_{1}}{2}\right), \\
& C_{m i j}^{k}=\left(i_{k}\right)^{2} \frac{k_{m}^{1}\left[\sin \left(k_{i}^{2} H_{2}\right)+k_{i}^{2} H_{2}\right]_{1}}{16 K_{m i j} \sin \left(k_{i}^{2} \frac{H_{2}}{2}\right)} \times \\
& \times \frac{\left[\sin \left(2 k_{j}^{3} H_{3}\right)+2 k_{j}^{3} H_{3}\right]}{\sin \left(k_{j}^{3} H_{3}\right)} \times \\
& \frac{m_{i j} \operatorname{sh}\left(\frac{m_{i j} H_{1}}{2}\right) \cos \left(k_{m}^{1} \frac{H_{1}}{2}\right)+k_{m}^{1} \operatorname{ch}\left(\frac{m_{i j} H_{1}}{2}\right) \sin \left(\frac{k H_{1}}{2}\right)}{\sin \left(k_{m}^{1} \frac{H_{1}}{2}\right)} \\
& K_{m i j}=\left(\left(k_{m}^{1}\right)^{2}+\left(k_{i}^{2}\right)^{2}+\left(k_{j}^{3}\right)^{2}\right), \\
& \varphi_{m i j}^{k}(x, t)=\cos \left(k_{m}^{1} x_{1}\right) \cos \left(k_{i}^{2} x_{2}\right) \cos \left(k_{j}^{3} x_{3}\right) \times \\
& \quad\left[\exp \left(b_{k}-a K_{m i j} t\right)-\frac{1}{b_{k}-a K_{m i j}}\right]
\end{aligned}
$$

Solution of thermal distribution equation for restoring regime was obtained:

$$
T_{r}(x, t)=g(x)+\sum_{i, j, m} C_{m i j}^{r} \varphi_{m i j}^{r}(x, t),
$$

where $g(x)=-\frac{b_{n}}{c_{n}}+\sum_{i, j, m} B_{m i j}^{r} \psi_{m i j}(x)$.

functions $\psi_{m i j}$ takes from form

$\psi_{m i j}=\exp \left(l_{m}^{1} x_{1}\right) \exp \left(l_{i}^{2} x_{2}\right) \exp \left(l_{j}^{3} x_{3}\right) ;$

$l_{m}^{1}, l_{i}^{2}, l_{j}^{3}$ - complex numbers such that

$\left(l_{m}^{1}\right)^{2}+\left(l_{i}^{2}\right)^{2}+\left(l_{j}^{3}\right)^{2}=b_{k} ;$

$B_{m i j}^{r}$ - are chosen in that way, that $g(x)$ satisfy

appropriate boundary conditions (we don't mention it here because of its complicated form),

$\varphi_{m i j}^{r}(x, t)=f_{m i j}^{r}(x, t) \cos \left(k_{m}^{1} x_{1}\right) \cos \left(k_{i}^{2} x_{2}\right) \cos \left(k_{j}^{3} x_{3}\right)$ $f_{m i j}^{r}= \begin{cases}\exp \left(\left(b^{k}-a K_{m i j}\right)\left(t-\tau_{k}\right)\right) & , t<t_{r}(x) \\ \exp \left(b^{k}\left(t_{r}(x)-t\right)-a K_{m i j}\left(t-\tau_{k}\right)\right), t \geq t_{r}(x)\end{cases}$

$t_{r}(x)$ - time of returning material to superconducting state at point in restoring regime $x$,

$t_{r}(x)=\left\{\begin{array}{lr}0, & T\left(x, \tau_{k}\right)<T_{c} ; \\ t \mid T(x, t)=T_{c}, & T\left(x, \tau_{k}\right)<T .\end{array}\right.$

Solution $T_{r}(x, t)$ satisfies equation (6) and boundary conditions (10-12) for any values of $C_{m i j}^{r}$.

For satisfaction boundary condition $(9,10,13)$, we assume that temperature at boundaries where superconductor is cooled by liquid nitrogen is lower than critical temperature.

$$
T(x)<T_{c} \text { at } x=\frac{H_{1}}{2}, \frac{H_{2}}{2}, H_{3} .
$$

Coefficients $C_{m i j}^{r}$ may be found from initial condition, using numerical integration.

$$
C_{m i j}^{r}=\frac{\int_{V}\left(T_{k}\left(x, \tau_{k}\right)-g(x)\right) \varphi_{m i j}^{r}\left(x, \tau_{k}\right) d x}{\int_{V}\left(\varphi_{m i j}^{r}\left(x, \tau_{k}\right)\right)^{2} d x},
$$

here $V$ - volume of superconductor film.

Restoring time $t_{r}(x)$ at any point $x$ can be found by iterative method.

\section{SIMULATION RESULTS}

Charachteristic of SFCL construction [10, 11], contact metal-superconductor $[8,11]$ and electrical regimes that were used for numerical simulation are presented in table 1.

Table 1. SFCL characteristics.

\begin{tabular}{|l|c|c|}
\hline $\begin{array}{c}\text { Construction } \\
\mathrm{mm}\end{array}$ & $\begin{array}{c}\text { Electrical } \\
\text { characteristic }\end{array}$ & \multicolumn{1}{c|}{$\begin{array}{c}\text { Contact } \\
\text { characteristic }\end{array}$} \\
\hline$H_{1}=50$ & $i_{n}=00.5 \frac{\mathrm{A}}{\mathrm{mm}^{2}}$ & $\rho_{c}=2.0 \cdot 10^{-14} \frac{\mathrm{Om}}{\mathrm{mm}^{2}}$ \\
\hline$H_{2}=10$ & $i_{k}=0.5 \frac{\mathrm{A}}{\mathrm{mm}^{2}}$ & $\lambda_{c}=295 \frac{\mathrm{J}}{\mathrm{mK}}$ \\
\hline$H_{3}=2-0.5$ & $\tau_{k}=0.002 \mathrm{sec}$ & $\beta_{c}=20 \frac{\mathrm{W}}{\mathrm{mm}^{2}}$ \\
\hline
\end{tabular}




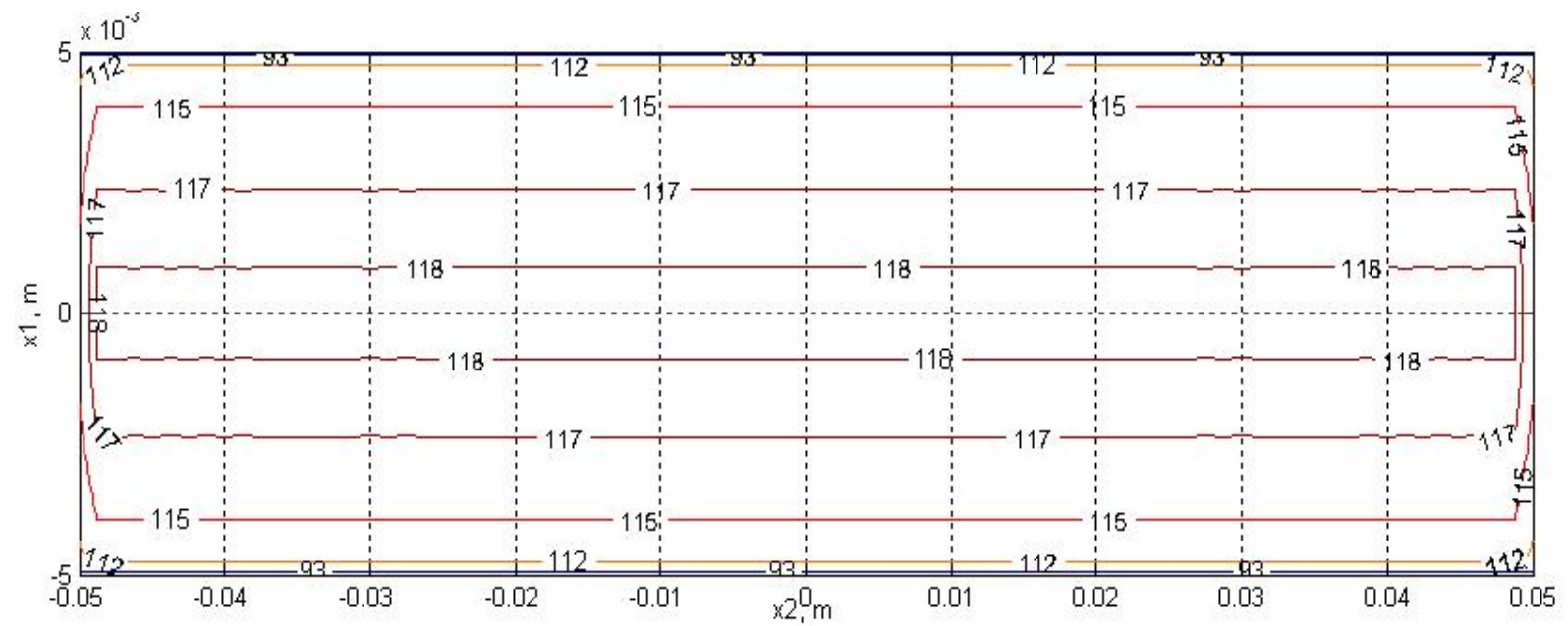

Fig. 2 - Temperature distribution at $\mathbf{t}=0.001$.

Temperature distribution in critical regime is presented on Fig. 2. For experiment chacharacteristic, the influence of contact metalsuperconductor is so insignificant that the thermal distribution in normal regime $T_{n}(x)$ is homogeneous and equals to the temperature of liquid nitrogen. Only when contact resistance is about $\rho_{c}=2.0 \times 10^{-4} \mathrm{Om} / \mathrm{m}^{2}$ it has influence on temperature distribution in normal regime.

Change of average temperature $T_{\text {aver }}(t)=\frac{1}{V} \int_{V} T(x, t) d x$ in superconductor film in critical and restore regime is shown on Fig 3 . Presented data are for same current density $i_{k}=0.5 \frac{\mathrm{A}}{\mathrm{mm}^{2}}$ in critical regime for different film height. For small film height $(H=0.2 \mathrm{~mm})$ temperature distribution can reach stable equilibrium in critical regime. Thick films are cooled slower than thin, so they reach high temperatures in critical regime. For this reason also thermal restore of thick superconducting films after current fault take more time than of thin films.

Dependence of film electrical resistance of time is presented on Fig. 4. Resistance rapidly increases at current fault start, as current density exceeds critical. At fault current resistance slowly increases due to heating. Changes of resistance at recovery regime depend on film height.

In critical regime resistance of film may vary for different load and film height. Heating in critical regime increase temperature of film and it has influence on electrical resistance. Maximum of the film resistance depends also on fault time (Fig. 5).

One of the most important characteristics of a SFCL is its recovery time after a fault. We define the recovery time as the time at which the difference of maximum temperature and liquid nitrogen is reduced to $1 / 100$. The sickness and the fault current are crucial factors for recovery time of SFCL film (Fig. 6).

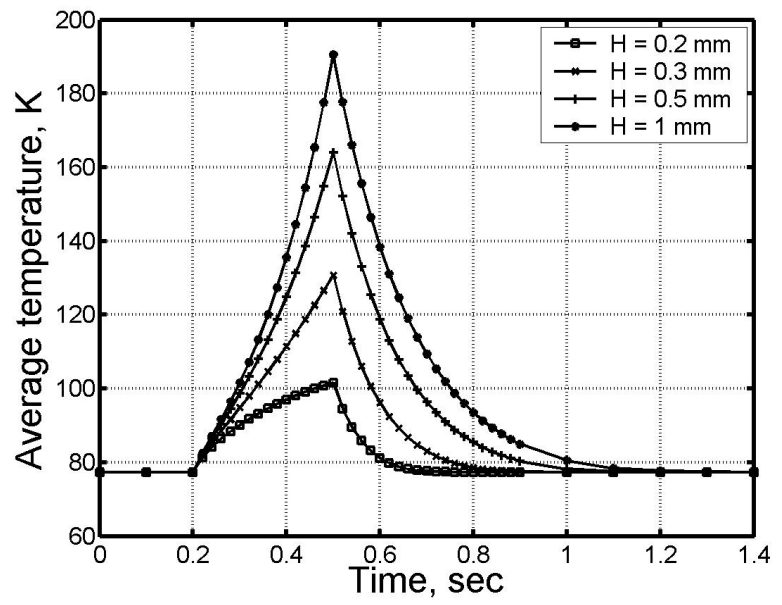

Fig. 3 - Average temperature of superconductor film for different film height.

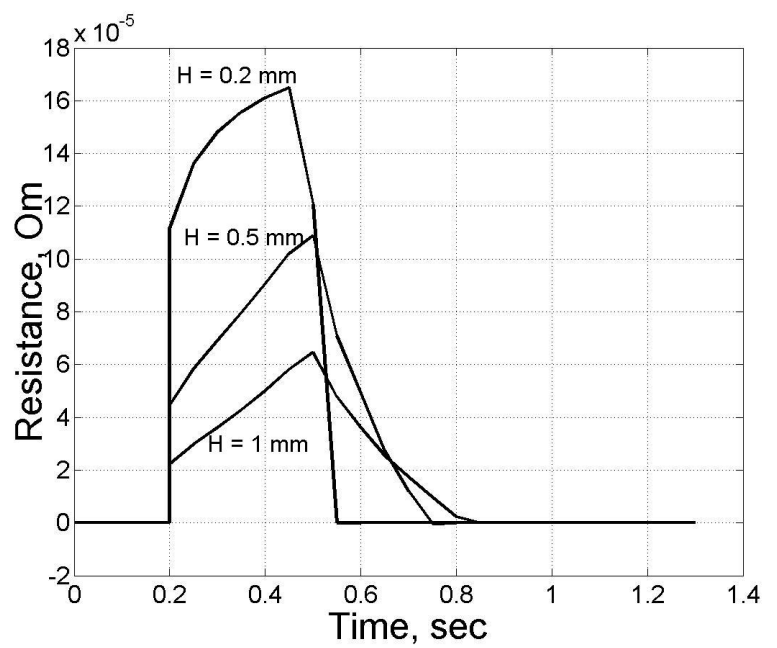

Fig. 4 - Resistance of superconducting film for different film height. 


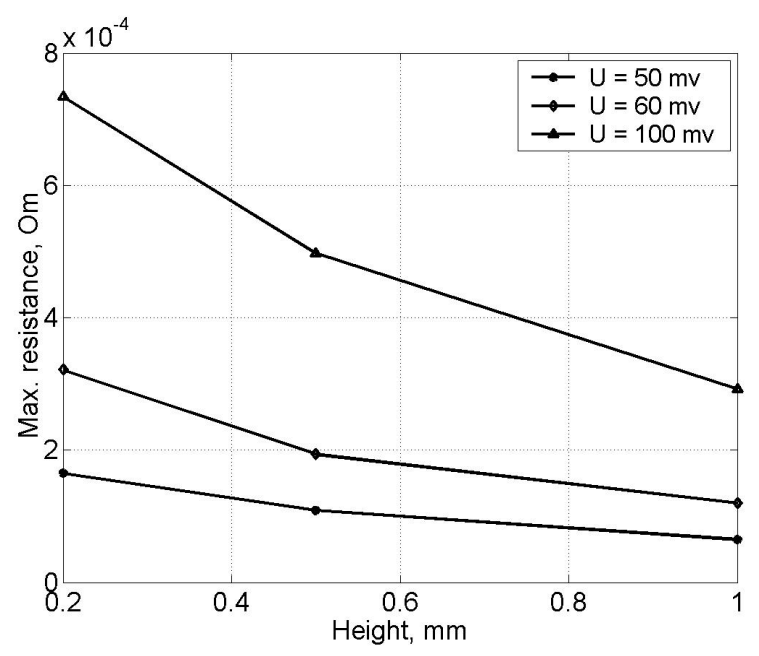

Fig. 5 - Maximum of film resistance in critical regime for different height and critical load.

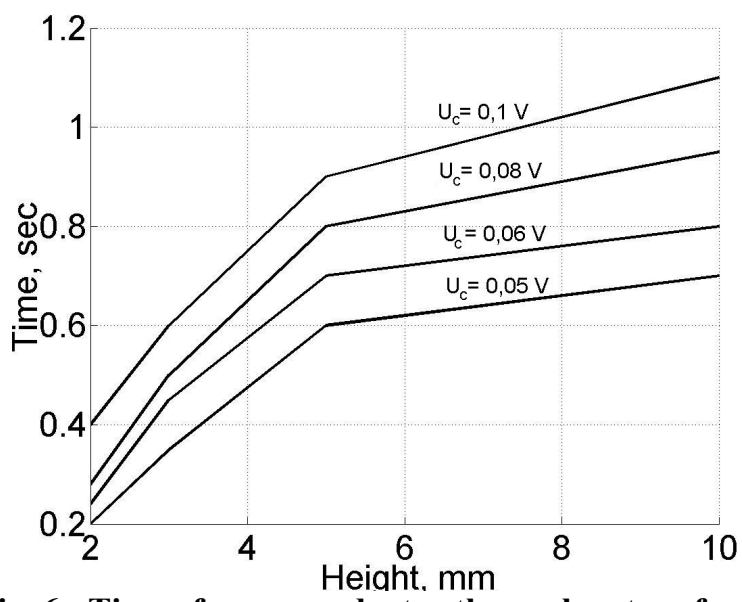

Fig. 6 - Time of superconductor thermal restore for different height and critical load.

\section{CONCLUSION}

Model of thermoelectrical processes in a resistive superconducting fault current limiter as thin film is presented. Analysis of normal, critical and restoring electrical regimes is performed.

An effective solution of the model is proposed. Thermal distribution and resistance of SFCL is analytically evaluated. Operational characteristics of SFCL were investigated. It was found that film construction parameters have critical influence for many characteristics of current limiter. It was also found that critical current and fault time have much influence on current limiting parameters, such as resistance at current fault and restore time.

\section{REFERENCES}

[1] R. Petranovic, A. M. Miri. "Lumped Network Model of a Resistive Type High Tc fault current limiter for transient investigations", International Conference on Power Systems Transients. - New Orleans, USA.-2003.-p.60-65.
[2] K. Kajikawa, K. Funaki, H. Hayashi, K. Terazono, and others. "Numerical study on fundamental properties of a resistive type fault current limiter with QMG bulk superconductor reinforced by metal bypass", IEEE Transactions on Applied Superconductivity.- 2004.- Vol. 14, No. 2 (2004.6), pp. 847-850.

[3] K. Kajikawa,Y. Takahashi, K. Enpuku, K. Funaki, and others. "Finite Element Analysis of Thermal and Mechanical Behaviour in Model Fault Current Limiter with QMG Bulk Superconductor", 19 International Conference on Magnet Technology Booklet.-Genoa, Italy.2005.-pp. 21-24-, No. MOA10P03.

[4] B. Zeimetz, K. Tanida, D.E. Eves, TJ. Chapman and others, "Thermal instability and current-voltage scaling in superconducting fault current limiters", Superconductor science technology. - 2004 - vol 17, .-p 657-662.

[5] A. Gurevich, "Thermal instability near planar defects in superconductors", Applied physics letters.- 26 March 2001.- volume 78, number 13.-p 1891-1893.

[6] R. A. Weller, "Computer Modeling of Superconducting Film Type Fault Current Limiters", IEEE Transactions on Applied Superconductivity.-1999.- Vol.9, No. 2 Pt1.pp.1377-1380

[7] J. Rymashewski, R. Pawlak. "Structural and electrical properties of metal-superconductor diffusion contact", MIXDES.-Wroclaw, Poland.June 2002.-p 283-286.

[8] J. Rymaszewski, "Numerical simulation of thermal phenomena in metal-superconductor junction", MIXDES.- Lodz, Poland.-June 2003.p 346-351.

[9] J. Leszcynski, J. Rymashewski, E. Korzeniewska. "Thermal phenomen in diffusion contact region of $\mathrm{Au} / \mathrm{bulk}$ superconductor YBaCuO", MIXDES.-Zakopane, Poland.-June 2001.-p 283-286.

[10] H. R. Semerad, H. Kinder, J. Grundmann, H. Mosebach, and others, "Current limiting properties of YBCO films on sapphire substrates", EUCAS 99.-Barcelona, Spain.-14.17.09 1999.p 30-32.

[11] S. Sugita, H. Ohsaki. "FEM Analysis of Current Limiting Characteristics of Superconducting Thin Film Current Limiting Device by the Current Vector potential Method", IEEE Transactions on Applied Superconductivity,.June 2003.-Vol. 13, Issue: 2.-p. 2020- 2023. 


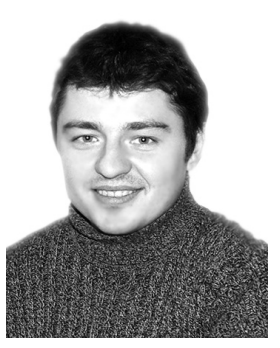

Pavlo Serdyuk, received his

Mater degree in Applied Mathematics in 2003 from Ivan Franko Lviv National University. Since 2003 he is PhD student at the department of Software Development of National University "Lviv Polytechnic". Areas of interests: $C A D$, thermal modeling, and numerical methods for mathematical physics.

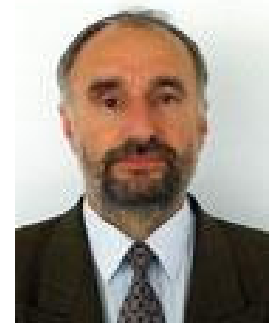

Dmytro Fedasyuk was born in 1955 in Ukraine. Doctor of technical sciences, Professor, Director of Institute of computer science and information technologies of Lviv Polytechnic National University, where works since 1980. The main scientific interests and researching fields: computer aided design of microelectronic systems, with particular emphasis on thermal design of 3-D ME's structure, development and application of models, algorithms and software for information technology. Graduated 3 candidates of technical sciences (Ph.D.). Author of 115 scientific publications including 2 monographs. Member of IEEE, Member of Shevchenko Science Society, Member of UFI in Ukraine. E-mail fedasyuk@polynet.Iviv.ua. 